\title{
On the Capacity Gain of Multi-Hop Cellular Networks with Opportunistic Networking and D2D: a Space-Time Graph-based Evaluation
}

\author{
Baldomero Coll-Perales, Member, IEEE, and Javier Gozalvez, Senior Member, IEEE
}

\begin{abstract}
Previous studies have shown that device-centric wireless technologies, including multi-hop cellular networks (MCNs) and device-to-device (D2D) communications, can increase the cellular network capacity. This study progresses the current state of the art by deriving (using space-time graphs) the upperbound capacity gains that can be further achieved when integrating efficiency-driven opportunistic networking into device-centric wireless networks. The increase is obtained without modifying the radio interface, and is not constrained to any particular radio interface.
\end{abstract}

Index Terms-Multi-hop cellular, opportunistic, 5G, devicecentric, D2D, space-time graph, capacity, UE-to-network relaying.

\section{INTRODUCTION}

5 G technologies will have to significantly increase the system capacity in order to handle the growth of mobile data traffic. This can be achieved through the combined use of new air interfaces, spectrum, and device-centric wireless technologies (including D2D and MCN) [1]. 3GPP has already standardized D2D and MCNs under R12 and R13 for proximity-based services. R14 is addressing further enhancements to the MCN concept (or UE-to-network relaying as referred to in 3GPP) for machine type communications. The potential of D2D and MCN has also sparked industrial innovations. For example, Vodafone introduced in 2016 its 4GFi technology that enables 4G devices to relay data for $2 \mathrm{G} / 3 \mathrm{G}$ devices using IEEE802.11 for the D2D.

Recent studies ([2]-[3]) suggest that device-centric wireless technologies could benefit from the integration of opportunistic networking. Opportunistic networking exploits the store, carry and forward capacity of mobile devices to establish communication links based on contact opportunities. Opportunistic networking has been generally proposed for disconnected or delay tolerant networks. However, [2] and [3] propose to utilize opportunistic networking together with MCN in order to establish links based on their efficiency and effectiveness and not just their presence. The integration of device-centric wireless technologies and efficiency-driven opportunistic networking is particularly relevant and viable if we consider that according to the 2016 Cisco Visual Networking Index, most of mobile data traffic is delay tolerant, with mobile video streaming representing the largest share. The studies reported in [2]-[3] have demonstrated that the integration of opportunistic networking and device-centric wireless technologies can reduce the energy consumption,

This work was supported in part by the Spanish Ministry Economy and Competitiveness and FEDER funds (TEC2014-5716-R), and the Generalitat Valenciana (GV/2016/049, and APOSTD/2016/049). increase the QoS, and offload cellular traffic. This study extends the current state of the art by investigating the upperbound system capacity gains that the integration of opportunistic networking and MCNs (referred to as opportunistic MCN in this paper) could provide over conventional cellular networks. To this aim, this paper proposes to model opportunistic MCN with space-time graphs, and defines an optimization framework to identify the configuration of opportunistic MCN that maximizes the capacity gains compared to conventional single-hop cellular networks.

\section{SPACE-Time Graph Model}

This study focuses on 2-hop uplink MCN communications ${ }^{1}$, where source nodes $(S)$ need to upload a message of size $F$ to the cellular base station $(B S)$ before a deadline of $T \mathrm{~s}$. To do so, $S$ must first establish a D2D link with a mobile relay $\left(R_{i}\right)$ that will then upload the information to the $B S$. This study considers that a D2D link can be established when devices are within a radio range $r_{D 2 D}$ and under line-of-sight (LOS). This ensures stable and high-throughput D2D links. This study considers the use of efficiency-driven opportunistic networking in the D2D and cellular links. In this case, opportunistic networking is utilized to search for and establish efficient D2D and cellular links, and not to handle disconnections. An efficient link is here defined as a link that reduces the cellular transmission time of a MCN connection, and hence increases the cellular network capacity. Other criteria could be considered.

Opportunistic networking has been commonly studied using graph theory as it provides the necessary tools to model the (pairwise) relations between nodes and their contact opportunities. Different types of network graphs can be used to represent the connectivity among mobile nodes [4]. Wireless graphs are time-varying graphs where edges between nodes indicate the presence of a wireless link. Wireless graphs are only modified when nodes move out of their communications range. Contact graphs are created by aggregating statistics from the dynamic wireless graphs (e.g. contact frequency or duration, or inter-contact time). Social graphs can partly solve the tractability problems of contacts graphs with a high number of nodes by defining sub-graphs (or communities) based on the social relations between nodes. All these graphs have been commonly utilized for networks that suffer disconnections, and

The authors are with the UWICORE laboratory, Universidad Miguel Hernandez de Elche, Spain (e-mail: \{bcoll, j.gozalvez\}@umh.es). ${ }^{1}$ The same process could be adapted to model downlink MCN. 
The final publication is available at: $\mathrm{http}$ ://ieeexplore.ieee.org/document/8010277/

assuming that a link is established as soon as two nodes get in contact with each other. This study considers a network without disconnections since all devices are always under cellular coverage. In this case, opportunistic networking is here utilized for establishing links between nodes, and between nodes and the infrastructure, when the most efficient transmission conditions are met. This study proposes to model these opportunistic MCN connections using space-time graphs. Space-time graphs create a dynamic sequence of graphs in order to represent the time evolution of the nodes' location and connectivity. Each graph represents a snapshot of the network topology, and it is assumed that the network conditions remain unchanged between snapshots. The snapshots are created every $\Delta t$ seconds in order to search for the conditions where efficiency-driven opportunistic networking can establish D2D and cellular links that minimize the cellular transmission time.

This study models 2-hop uplink MCNs as a sequence of graphs $g=\left\{G_{1 \Delta t}, \ldots, G_{t \Delta t} \ldots, G_{T \Delta t}\right\}$, where $G_{t \Delta t}=\left\{v_{t \Delta t}, e_{t \Delta t}\right\}$ is the graph representing the network snapshot at time $t \Delta t$. $v_{t \Delta t}=(S \cup$ $B S \cup r$ ) denotes the nodes of the graph, and $r$ refers to the set of potential mobile relays between $S$ and the $B S . e_{t \Delta t}=\left\{\left(S, R_{i}\right),\left(R_{i}\right.\right.$, $\left.B S) \mid \forall R_{i} \in r ; S, r, B S \in v_{t \Delta t}\right\}$ denotes the edges that make up the network graph. An $\left(S, R_{i}\right)$ edge indicates the presence of a $S-R_{i}$ D2D link $\left(R_{i} \in r\right)$. After an $\left(S, R_{i}\right)$ edge is created, an $\left(R_{i}, B S\right)$ edge is established for all subsequent snapshots. The $\left(R_{i}, B S\right)$ edge represents the presence of a $R_{i}-B S$ cellular link. Fig. 1 represents an space-time graph built to model 2-hop uplink opportunistic MCN connections. The objective of this study is to investigate the maximum capacity gains that could be achieved with opportunistic MCN compared to conventional single-hop cellular communications. Then, the space-time graph models all possible D2D and cellular links in order to identify the ones that minimize the cellular transmission time and hence maximize the capacity gains. The graph models the integration of opportunistic networking into D2D and cellular links. As a result, the graph considers the possibility that $S$ transmits the message of size $F$ to another device through a D2D link, and this device uploads the information to the $B S$ later even if the D2D link with $S$ is broken. Fig. 1 represents different snapshots of the space-time graph of a source node $S$ that has a message to transmit, and $T$ seconds to do so. At $1 \Delta t$, $S$ identifies as potential relays $R_{1}, R_{2}$ or $R_{3}$. New D2D connections can be identified at different time instants. At $2 \Delta t$, the graph models the possibility to establish a cellular link between the identified relays $\left\{R_{1}, R_{2}, R_{3}\right\}$ and the $B S$. The D2D and cellular transmissions do not need to start as soon as the links are identified, but at any point in time as long as the message is completely received by the $B S$ before $T$. At $2 \Delta t$, a new possible D2D connection between $S$ and $R_{4}$ is identified, and the D2D connection between $S$ and $R_{l}$ breaks. The cellular link between $R_{l}$ and the $B S$ is maintained as the message could have been transmitted from $S$ to $R_{l}$ before the D2D connection broke. In this case, $R_{l}$ could store the message and later upload it to the $B S$. The $R_{4}-B S$ cellular link can be established from $3 \Delta t$. At $3 \Delta t$, the D2D links between $S$ and $R_{2}$, and between $S$ and $R_{3}$ break. However, the two devices maintain the cellular link to the $B S$ for the same reason as indicated for $R_{l}$.

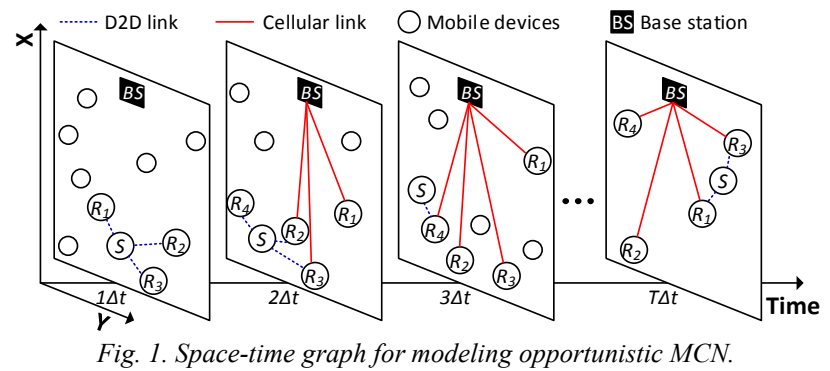

\section{OPTIMIZATION FRAMEWORK}

The constructed space-time graph models all possible D2D and cellular links that will allow a source node to upload a message to the $B S$ before the deadline $T$ using opportunistic MCN. The graph has then been used to identify the D2D and cellular links of the opportunistic MCN connection that would minimize the cellular transmission time, and hence result in the largest capacity gain. To this aim, we propose the following optimization framework:

$$
\begin{gathered}
\text { o.f: } \min \left(N_{R_{i}}\right)=\min \left(\sum_{x=2 \Delta t}^{\mathrm{T} \Delta t} c_{x}^{\left(R_{i}, B S\right)}\right) \\
\text { s.t.: } \sum_{x=1 \Delta t}^{\mathrm{T} \Delta t-\Delta t} d_{x}^{\left(S, R_{i}\right)}=t_{D 2 D} / \Delta t \\
\sum_{x=2 \Delta t}^{\mathrm{T} \Delta t} c_{x}^{\left(R_{i}, B S\right)} \cdot t h r_{R_{i}}(x) \cdot \Delta t \geq F \\
\sum_{x=2 \Delta t}^{X} c_{x}^{\left(R_{i}, B S\right)} \cdot t h r_{R_{i}}(x) \cdot \Delta t \leq \sum_{x=1 \Delta t}^{X-\Delta t} d_{x}^{\left(S, R_{i}\right)} \cdot \Gamma, \forall X \in[2 \Delta t \ldots \mathrm{T} \Delta t]
\end{gathered}
$$

where $N_{R i}$ represents the cellular transmission time from $R_{i}$ to the $B S . d_{x}{ }^{(S, R i)}$ and $c_{x}{ }^{(R i, B S)}$ are binary variables used to indicate when D2D and cellular transmissions actually take place between $S$ and $R_{i}$, and between $R_{i}$ and the $B S$, respectively. The variables are equal to one when the transmission takes place, and equal to zero otherwise. The D2D transmissions can only take place when $S$ and $R_{i}$ are under LOS and at a distance smaller than $r_{D 2 D}$. The variable $c_{x}{ }^{(R i, B S)}$ can only be equal to one if $R_{i}$ has been previously in contact with $S$, i.e. if $R_{i}$ has been under LOS conditions with $S$ at a distance smaller than $r_{D 2 D} . x$ $\epsilon[1 \Delta t, \ldots T \Delta t-\Delta t]$ for $d_{x}^{(S, R i)}$, while $x \in[2 \Delta t, \ldots T \Delta t]$ for $c_{x}^{(R i, B S)}$ because $R_{i}$ needs to have received (at least part of) the message from $S$ before being able to start the upload to the $B S$.

The objective function (1) is subject to a set of constraints. Constraint (2) ensures that the selected mobile relay $R_{i}$ has a D2D link with $S$ at least for $t_{D 2 D}$ seconds (or during $t_{D 2 D} / \Delta t$ events in the space-time graph; these events do not need to be consecutive in time as opportunistic networking is integrated with D2D). $t_{D 2 D}$ represents the duration of the D2D transmission from $S$ to $R_{\mathrm{i}}$. Constraint (3) ensures that the message of size $F$ is completely transmitted from $R_{i}$ to the $B S$ before $T$. $t h r_{R i}(x)$ represents the uplink cellular throughput experienced by $R_{i}$ at time instant $x$. Constraint (4) guarantees that the $\left(R_{i}, B S\right)$ cellular link does not transmit more information than what is received through the $\left(S, R_{i}\right) \mathrm{D} 2 \mathrm{D}$ link. Г represents the data transmitted in the $\mathrm{D} 2 \mathrm{D}$ link during $\Delta t$ (i.e. $\left.\mathrm{I}=\Delta t \cdot F / t_{D 2 D}\right)$.

The optimization framework is executed for all mobile devices which have been in contact with $S$ (i.e. $\forall R_{i} \in R$ ) during 
The final publication is available at: $\mathrm{http}$ ://ieeexplore.ieee.org/document/8010277/

$[1 \Delta t, \ldots T \Delta t-\Delta t]$. Then, the configuration of opportunistic MCN that minimizes the cellular transmission time is identified as:

$\left[c_{x}^{\left(R^{*}, B S\right)}, d_{x}^{\left(S, R^{*}\right)}\right]=\arg \min _{\forall R_{i} \in R}\left\{N_{R_{i}}\left(\alpha_{x}^{\left(R_{i}, B S\right)}, \beta_{x}^{\left(S, R_{i}\right)}, t_{D 2 D}, t h r_{R_{i}}, F, T\right)\right\}(5)$

This configuration is defined by the selected relay $R^{*}$, and the time instants at which the D2D (from $S$ to $R^{*}$, i.e. $d_{x}^{\left(S, R^{*}\right)}$ ) and cellular (from $R^{*}$ to the $B S$, i.e. $c_{x}{ }^{\left(R^{*}, B S\right)}$ ) transmissions take place. Finally, the identified configuration is used to compute the minimum cellular transmission time $(N)$ that can be achieved with opportunistic $\mathrm{MCN}$ as:

$$
N=\sum_{x} c_{x}^{\left(R^{*}, B S\right)}=\min _{\forall R_{i} \in R}\left(N_{R_{i}}\right)
$$

\section{EVAluATION ENVIRONMENT}

The evaluation environment follows 3GPP guidelines and models for the simulation of outdoor urban scenarios (3GPP TR36.873 and [6]). The study considers a Manhattan-like scenario with $\{5 \times 5,8 \times 8,11 \times 11\}$ blocks. The width of buildings and streets is set equal to $89 \mathrm{~m}$ and $11 \mathrm{~m}$ respectively. These settings are selected to analyze the performance under various heterogeneous outdoor deployments characteristic of urban environments. The $B S$ is located at the center of the scenario, and provides coverage to the complete scenario. The cell radius is then equal to $\{350,560,770\} \mathrm{m}$ for these scenarios respectively. Mobile devices are uniformly distributed, and move at $2 \mathrm{~m} / \mathrm{s}$. At intersections, the devices have the same probability of turning right, left or continue straight. Different densities of nodes have been modelled following the guidelines in [6] for the test case "dense urban information society (duis)". The spatial density of mobile devices $(\lambda)$ within a cell has been set equal to $\{0.25,0.5\} \cdot \lambda_{\text {duis }}{ }^{2} . \lambda_{\text {duis }}$ is the spatial density of pedestrians in [6], and is equal to 8.500 users $/ \mathrm{km}^{2}$. The study does not focus on any particular traffic service, but considers that source nodes need to upload a file of size $F=30 \mathrm{Mb}$ before a deadline $T=\{30,100,220\}$ s. $T 30,100$ and 220s correspond to the time conventional single-hop cellular communications need to complete the transmission of $F$ at the border of cells with $\{350,560,770\} \mathrm{m}$ radius respectively.

The cellular connection to the $B S$ is an LTE connection at $2 \mathrm{GHz}$. The system bandwidth is set to $10 \mathrm{MHz}$, and the cellular transmissions utilize 6 physical resource blocks. The cellular propagation losses are modelled using the 3D urban macrocellular (3D-UMa) channel model reported in 3GPP TR36.873. Log-normal fading with standard deviation $\sigma_{S F}=4 \mathrm{~dB}$ (LOS) and $\sigma_{S F}=6 \mathrm{~dB}$ (NLOS) is also included. The LTE uplink throughput is estimated using the SNR-BER curves reported in [7] for different CQIs (the target BER is 0.1), and the tables in 3GPP TS36.213 that map CQI values and transmission modes to the associated transport block index $\left(i_{T B S}\right) . i_{T B S}$ varies from 0 to 26 with the highest values associated to the highest transmission modes (i.e. the combinations of modulation and coding schemes resulting in the highest data rates) and larger transport block sizes.

$S$ communicates with $R$ using $802.11 \mathrm{~g}$ at $2.4 \mathrm{GHz}$. 3GPP considers both 802.11 and LTE for D2D (or sidelink as referred to in 3GPP) communications. The empirical measurements in [8] showed that $802.11 \mathrm{~g}$ uses its highest data rate (54Mbps) up to a maximum distance of $80 \mathrm{~m}$ under LOS. $r_{D 2 D}$ has then been set equal to $80 \mathrm{~m}$ in this study. Radio transmission errors and medium access overhead (or channel efficiency as defined in [8]) result in an $802.11 \mathrm{~g}$ throughput that varies between 15 and $30 \mathrm{Mbps}$ for distances up to $80 \mathrm{~m}$ under LOS when using the $54 \mathrm{Mbps}$ data rate. We assume the worst-case scenario, and consider that all D2D transmissions experience a $15 \mathrm{Mbps}$ throughput. The time needed to transmit a file of size $F=30 \mathrm{Mb}$ at $15 \mathrm{Mbps}$ over a D2D link $\left(t_{D 2 D}\right)$ has hence been set to $2 \mathrm{~s}$.

The scenario is simulated in Matlab. $\Delta t$ is set equal to $1 \mathrm{~s}$ as a compromise between tractability and accuracy. A minimum of 10.000 experiments have been conducted for each combination of parameters to guarantee that the standard error of the mean is below $0.5 \%$. A user $S$ within the cell is randomly selected in each experiment to solve the optimization framework (1)-(6) using exhaustive search. The performance experienced by $S$ using opportunistic MCN is compared against that experienced by $S$ (same position and time instant) using conventional singlehop cellular communications where $S$ directly transmits the information to the $B S$ ( $\mathrm{SH}$ mode). To this aim, we obtain a value of $N$ (using (6)) and $N_{S}$ for each experiment. $N_{S}$ is the cellular transmission time needed by $S$ to upload the file using the $\mathrm{SH}$ mode. In this mode, the cellular transmission from $S$ to the $B S$ starts at time instant $1 \Delta t$ and cannot be paused. We then compute $\gamma$ that represents the average percentage reduction of the cellular transmission time that opportunistic MCN achieves with respect to the $\mathrm{SH}$ mode. $\gamma$ is computed as the average value of $100 *\left(1-N / N_{S}\right)$ over all the experiments conducted for each combination of parameters. The capacity gain is computed as $100 \cdot(\gamma /(100-\gamma))$, and represents how many more opportunistic $\mathrm{MCN}$ transmissions than $\mathrm{SH}$ ones can be served with the same cellular channel occupancy.

\section{CAPACITY ANALYSIS}

The proposed space-time graph and optimization framework are utilized in this section to identify the upper-bound of the capacity gains that can be obtained with the utilization of opportunistic MCN. Fig. 2 shows the average capacity gain (in $\%)$ that opportunistic MCN can achieve with respect to $\mathrm{SH}$. Fig. 2 shows that the minimum capacity gain for all scenarios is $36.8 \%$ (cell radius $=350 \mathrm{~m}, T=30 \mathrm{~s}$ ); this corresponds to a reduction of the cellular transmission time of $26.9 \%$.

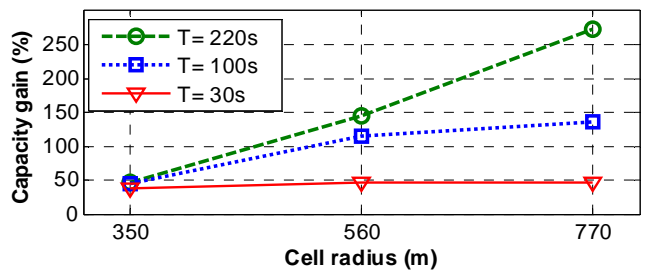

Fig. 2. Capacity gain $\left(\lambda=0.5 \cdot \lambda_{\text {duis }}\right)$.

Fig. 2 shows that the capacity gains obtained with opportunistic MCN augment with $T$ and the cell size. For example, opportunistic MCN increases the capacity by $47.1 \%$ 
The final publication is available at: $\mathrm{http}$ ://ieeexplore.ieee.org/document/8010277/

when $T$ is equal to $30 \mathrm{~s}$ and the cell size is $560 \mathrm{~m}$. The capacity gain augments to $143.9 \%$ when $T$ is set equal to $220 \mathrm{~s}$. As the service deadline $T$ increases, opportunistic MCN can find better combinations of D2D and cellular links to reduce the cellular transmission time. The cell size has also a significant impact. Larger cell sizes degrade the QoS of conventional single-hop cellular communications, in particular for users far away from the $B S$. These users significantly benefit from opportunistic MCN that considerably improves their link budget. For example, opportunistic MCN increases the capacity by $45.7 \%$ when the cell size is $350 \mathrm{~m}$ and $T$ is equal to $220 \mathrm{~s}$. The capacity increases by $273.1 \%$ when the cell size augments to $770 \mathrm{~m}$. The results reported in Fig. 2 correspond to average values across the cell area. The impact of opportunistic MCN augments when the source node is not close to the $B S$ (in particular, at distances higher than $150 \mathrm{~m}$ ). For example, opportunistic MCN reduces the cellular transmission time by $20 \%$ when the source node is $250 \mathrm{~m}$ away from the $B S(T=220 \mathrm{~s}$, cell size $=770 \mathrm{~m})$. This value augments to $82 \%$ when the source node is $550 \mathrm{~m}$ away from the $B S$. Similar trends as those reported in Fig. 2 have been observed for lower spatial densities of nodes, in particular for $\lambda=0.25 \cdot \lambda_{\text {duis }}$. However, the benefits of opportunistic MCN augment with $\lambda$ because of the higher contact opportunities between nodes. The impact of $\lambda$ reduces when $T$ increases.

Opportunistic MCN can reduce the cellular transmission time since it is able to identify favorable communication conditions under which to perform the D2D and cellular transmissions. In the conducted evaluations, the cellular transmissions within opportunistic MCN connections always experienced higher CQI levels than SH. High CQI values are associated to high modulation and coding schemes, and consequently higher throughput levels. For example, $99 \%$ of the cellular transmissions within opportunistic MCN connections experienced the highest CQI levels when $T=100 \mathrm{~s}$ and the cell radius was equal to $350 \mathrm{~m}$. This value decreased to only $18 \%$ for the SH mode.

Fig. 3 compares the capacity gain achieved with opportunistic MCN ('Opp-MCN' in Fig. 3) to that obtained with other communication modes. The opportunistic cellular mode ('Opp-cellular' in Fig. 3) refers to the case in which source nodes do not use mobile relays, and directly transmit the information to the $B S$ using a cellular connection that integrates the efficiency-driven opportunistic networking paradigm. In the MCN mode, $S$ transmits the information to the $B S$ using a MCN connection that does not utilize opportunistic networking at the D2D and cellular links. Similarly to the opportunistic MCN mode, the selected mobile relay has to be in LOS conditions with $S$ and within a radio range $r_{D 2 D}$. The MCN mode requires selecting the mobile relay at time $1 \Delta t$, and starting the cellular transmissions from the selected mobile relay to the $B S$ at time $2 \Delta t$. For a fair comparison with the opportunistic MCN mode, we have designed optimization processes similar to that reported in (1)-(6) for the opportunistic cellular and MCN modes. These processes have been adapted to the characteristics of these two modes, but also seek to identify the time instants at which the cellular transmissions should take place in order to minimize the cellular transmission time. In particular, the optimization framework defined for the opportunistic cellular mode includes equations (1) and (3) with $x \in[1 \Delta t, \ldots T \Delta t]$ for $c_{x}{ }^{(S, B S)}$, but does not include the term $d_{x}{ }^{(S, R i)}$ as the mode does not utilize D2D transmissions. The optimization framework for the MCN mode includes the equations (1)-(4) with $x \in[1 \Delta t, 2 \Delta t]$ for $d_{x}{ }^{(S, R i)}$, and an additional constraint ( $\left.\sum_{x=2 \Delta t}^{X} c_{x}^{\left(R_{i}, B S\right)} \cdot t h r_{R_{i}}(x) \cdot \Delta t>\sum_{x=1 \Delta t}^{X-\Delta t} c_{x}^{\left(R_{i}, B S\right)} \cdot t h r_{R_{i}}(x) \cdot \Delta t, \quad \forall X \in[2 \Delta t \ldots \mathrm{T} \Delta t]\right)$, to indicate that the cellular transmissions from the selected mobile relay to the $B S$ have to start at $2 \Delta t$ and cannot be paused. Using their respective frameworks, we also derive the minimum cellular transmission times $(N)$ for the opportunistic cellular and MCN modes (following eq. (6)), and compute their capacity gains with respect to the $\mathrm{SH}$ mode following the same procedure as defined for the opportunistic MCN mode. Fig. 3 demonstrates that the large capacity gains achieved by opportunistic MCN result from the combined use of opportunistic networking and MCN.

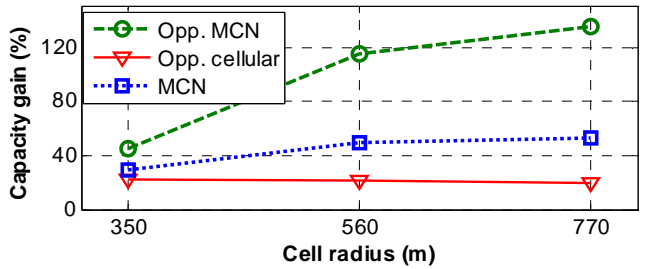

Fig. 3. Capacity gain $\left(\lambda=0.5 \cdot \lambda_{\text {duis, }} T=100 \mathrm{~s}\right.$; similar trends for $\left.T=(30,220) \mathrm{s}\right)$.

\section{CONCLUSIONS}

This study has investigated the capacity gains that can be obtained from the utilization of opportunistic networking and MCN. To do so, the study models opportunistic MCN connections using a space-time graph, and proposes an optimization framework for minimizing the cellular transmission time. The results show that opportunistic MCN can significantly increase the cellular capacity (by up to $273 \%$ under the evaluated conditions). The gains are particularly relevant as the cell size or service deadline increase. Opportunistic MCN can increase the capacity without modifying the radio interface, and the increase is not constrained to any radio interface. This paves the new way for further gains as new $5 \mathrm{G}$ radio interfaces are introduced.

\section{References}

[1] F. Boccardi, et al., "Five disruptive technology directions for 5G", IEEE Commun. Mag., vol. 52, no. 2, pp. 74-80, Feb. 2014.

[2] B. Coll-Perales, et al., "Context-aware opportunistic networking in multihop cellular networks", Ad Hoc Netw., vol. 37, pp. 418-434, Feb. 2016.

[3] V. Sciancalepore, et al., "Offloading Cellular Traffic through Opportunistic Communications: Analysis and Optimization", IEEE J. Sel. Area. Comm., vol. 34, no. 1, pp. 122-137, Jan. 2016.

[4] M. Schurgot, et al., "Beyond traditional DTN routing: social networks for opportunistic communication", IEEE Commun. Mag., vol. 50, no. 7, pp. 155-162, July 2012

[5] Y. Li, et al., "A Dynamic Graph Optimization Framework for Multihop Device-to-Device Communications Underlaying Cellular Networks", IEEE Wirel. Commun., vol. 21, no. 5, pp. 52-61, Oct. 2014.

[6] METIS Deliverable D.6.1. "Simulation guidelines". Oct. 2013.

[7] J. Blumenstein, et al., "Simulating the long term evolution uplink physical layer", Proc. of IEEE ELMAR, Sept. 2011, Zadar (Croatia).

[8] B. Coll-Perales, et al., "Empirical Performance Models for P2P and Two Hops Multi-hop Cellular Networks with Mobile Relays", Proc. of the ACM PM2HW2N, 3-8 Nov. 2013, Barcelona (Spain). 